\title{
Cartas de control para monitorear variables multinomiales
}

Recibido:

Noviembre 6 de 2013

Aceptado:

Marzo 25 de 2014

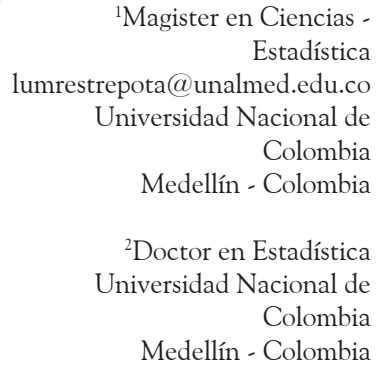

\section{Control charts for monitoring multinomial variables}

\section{Resumen}

Antecedentes: La carta de control como herramienta de monitoreo de la calidad de un producto, permite estudiar la estabilidad de los procesos en el tiempo, contrastando dos hipótesis, una que expresa que el proceso se encuentra en estado estable y otra que lo niega. Su utilización ha sido masiva para variables continuas más no para variables categóricas, motivo por el cual es imperante el diseño de tales herramientas para ese tipo de variables. Objetivo: Proponer dos (2) cartas de control para procesos con variables multinomiales basadas en el valor-p resultado de la prueba de homogeneidad de proporciones, empleando la transformación chi cuadrado para variables uniformes y la aproximación Wilson - Hilferty para variables chi cuadrado. Métodos: El desempeño de las cartas propuestas es estimado vía simulación considerando un proceso en Fase II y considerando incrementos en la primera categoría de 2\%, 4\% y 6\% en la etapa de control. Resultados: La carta de control multinomial usando aproximación WilsonHilferty para variables chi cuadrado, provenientes de la transformación del valor-p, presenta un desempeño deficiente comparado con las cartas de control usando valor-p y usando transformación chi cuadrado al valor-p, pues tienen menor habilidad para detectar cambios pequeños. Conclusión: Proponemos dos cartas de control para monitorear variables multinomiales y, una vez estudiadas vía simulación, con base en la Longitud de corrida promedio (ARL) y la probabilidad de rechazar la hipótesis nula de igualdad de proporciones, se recomienda el uso de la carta de control usando valor-p, o equivalentemente, de la carta de control usando transformación chi cuadrado del valor-p. ISSN 0122-820X

\author{
Luz Marcela Restrepo-Tamayo ${ }^{1}$, Juan Carlos Correa-Morales ${ }^{2}$
}

Forma de citar: L. M. Restrepo, J. C. Correa, "Cartas de control para monitorear variables multinomiales”, Respuestas, vol. 19, no. 2, pp. 93-100, 2014.
\end{abstract}


product, allows to study the stability of processes over time, contrasting two hypothesis, which states that the process is in stable condition and the other denies it. Its use has been massive for continuous variables but not for categorical variables, why it is imperative to design such tools for such variables. Objective: To propose two (2) control charts for variables multinomial processes based on the $\mathrm{p}$-value test result for homogeneity of proportions using the chi square test for uniform processing variables and approximation Wilson - Hilferty for variables chi square. Methods: The performance of proposed charts via simulation is estimated considering a Phase II process and considering the first category increments of $2 \%$, $4 \%$ and $6 \%$ in the control stage. Results: The multinomial control chart using Wilson-Hilferty approximation for variables chi square, from the transformation of value-p, has poor performance compared to the control charts using $\mathrm{p}$-value processing and using chi-square $\mathrm{p}$-value, as they have less ability to detect small changes. Conclusion: We propose two control charts to monitor multinomial variables and once studied via simulation, based on the average run length (ARL) and the probability of rejecting the null hypothesis of equal proportions, we recommend the control chart using value-p, or equivalently, the control chart processing using chi square p-value.

Keywords: control chart, multinomial distribución, homogeneity test, p-value.

\section{Introducción}

Las cartas de control hacen parte de un conjunto de herramientas llamado Control Estadístico de Procesos (CEP), que permite, bajo el esquema de control de calidad, garantizar productos adecuados para el uso, mediante el análisis de la variación del proceso que los genera [1]. Las más comunes son las cartas de control de Shewhart basadas en la distribución normal y, de manera general, para variables aleatorias continuas utilizando transformaciones y aproximaciones, evidenciando además su implementación de manera intensiva. Para variables discretas no se tiene el mismo panorama, pues éstas no brindan tanta información como aquellas [2], pero han sido de gran utilidad para monitorear servicios y evaluar aspectos de apariencia de producto terminado. Dado este panorama, nos interesa de manera particular,

implementar una carta de control que permita identificar cambios cuando se tienen más de dos categorías, esto es, para procesos con variables multinomiales.

Considere una serie de $n$ ensayos idénticos e independientes, en cada uno de los cuales solamente uno de $c$ eventos exclusivos $E_{1}, E_{2}, \ldots, E_{c}$ puede ser observado con probabilidades $\pi_{1}, \pi_{2}, \ldots, \pi_{c}$ respectivamente, donde $\pi_{1}+\pi_{2}+\cdots+\pi_{c}=1$ . Sea $N_{1}, N_{2}, \ldots, N_{c}$ variables aleatorias que representan el número de ocurrencias de los eventos $E_{1}, E_{2}, \ldots, E_{c}$ respectivamente, en esos $n$ ensayos, con $\sum_{i=1}^{c} N_{i}=n$. Entonces la distribución conjunta de $N_{1}, N_{2}, \ldots, N_{c}$ está dada por (1) [3].

$$
p\left(n_{1}, n_{2}, \ldots, n_{c}\right)=\left(\frac{n !}{n_{1} ! n_{2} ! \ldots n_{c} !}\right) \pi_{1}^{n_{1}} \pi_{2}^{n_{2}} \ldots \pi_{c}^{n_{c}}
$$


Para controlar procesos con este tipo de variables, Marcucci [4] plantea una propuesta utilizando el estadístico $\chi 2$ de Pearson. Laviolette [5] utiliza un enfoque bayesiano que utiliza la distribución Dirichlet como distribución a priori de la distribución multinomial concentrándose en categorías no conformes, mientras que Shiau, Chen y Feltz [6] se enfocan tanto en categorías conformes como no conformes. Woodall, Tsui y Tucker [7] presentan una revisión de cartas de control basadas en estadística difusa para datos categóricos, y Spanos y Chen [8] utilizan el concepto de regresión logística para monitorear una variable multinomial en la fabricación de semiconductores. Duran [9] propone el llamado método p-tree que transforma el proceso multinomial en varias subetapas binarias independientes, sin limitaciones con respecto al número de categorías que tenga el proceso. Raz y Wang [10] presentan dos propuestas basadas en la teoría de conjuntos difusos y en el uso de subconjuntos difusos para modelar los términos categóricos usados para describir la calidad del producto. Taleb y Limam [11] comparan el enfoque difuso y el enfoque probabilístico mediante la longitud promedio de la corrida (ARL) asumiendo muestras bajo control. Taleb, Limam y Hirota [12] plantean la utilización de un estadístico similar al de $T^{2}$ de Hotelling's basado en valores representativos de conjuntos difusos, y también proponen la utilización del estadístico $W^{2}$ basado en la combinación lineal de variables chi cuadrado dependientes usando la aproximación Satterthwaite's. Finalmente, Taleb [13] presenta cartas de control para múltiples variables multinomiales empleando una aproximación probabilística basada en el estadístico $\chi 2$ de Pearson y cuatro propuestas basadas en conjuntos difusos, empleando un método intra-difuso y otro método interdifuso, y Li y Tsung [14] proponen cartas de control para múltiples variables binomiales y múltiples variables Poisson basadas en la tasa de falso descubrimiento (FDR), que se utiliza para cuantificar la proporción esperada de rechazos erróneos.

Con base en la revisión anterior, proponemos dos cartas de control para procesos con variables multinomiales, teniendo en cuenta que para esta distribución se requieren tamaños de muestra grandes y por lo tanto sólo aplicarán para procesos con volúmenes altos de producción. Estas propuestas están basadas en el valor-p resultado de la prueba de homogeneidad de proporciones para datos multinomiales (o prueba chi cuadrado de bondad de ajuste) y, se analiza su desempeño con base en la sensibilidad para detectar cambios vía simulación usando el paquete estadístico R.

\section{Cartas de control propuestas}

Partiendo del hechode que una cartade control en Fase II prueba una hipótesis de igualdad con respecto a un valor especificado repetidamente en diferentes puntos del tiempo [2], es de interés probar la hipótesis de igualdad de proporciones muestra a muestra con respecto a un vector de proporciones nominal. Esto es, para comparar las proporciones de la muestra $i$ con las proporciones nominales dadas por un vector nominal $v=\left(\pi_{i 1}^{(0)}, \pi_{i 2}^{(0)}, \ldots, \pi_{i c}^{(0)}\right)$ las pruebas de hipótesis estarán dadas en (2)-(3).

$$
\begin{array}{r}
H_{0}: \pi_{i 1}=\pi_{i 1}^{(0)}, \pi_{i 2}=\pi_{i 2}^{(0)}, \ldots, \pi_{i c}=\pi_{i c}^{(0)} \\
H_{1}: \exists\left(\pi_{i j} \neq \pi_{i j}^{(0)}\right), j=1,2, \ldots, c
\end{array}
$$

Cuando un proceso con variables multinomiales está bajo estudio, es importante analizar el tamaño de muestra con respecto al número de categorías y a la probabilidad de ocurrencia de cada una de ellas, ya que un número grande de categorías puede conducir a proporciones poco representativas dentro del análisis, por lo anterior, las categorías del proceso a ser monitoreadas deben establecerse de acuerdo a su naturaleza y al tipo de análisis que se pretenda llevar a cabo.

ulio-Diciembre 2014

ISSN 0122-820X

P: $93-100$

Para las siguientes cartas de control propuestas, los límites de control están relacionados con el vector de proporciones nominal, el cual se deberá construir adaptativamente, a partir de los datos históricos del proceso. Una vez establecidos los límites de control provisionales, se debe identificar la presencia 
No. 2

Julio-Diciembre 2014 ISSN 0122-820X

PP: 93-100 de patrones de comportamiento no aleatorio, de tal manera que ante la presencia de causas asignables será necesario intervenir el proceso, tomar nuevas muestras, recalcular el vector de proporciones nominal y proceder a elaborar nuevamente la carta de control.

\subsection{Propuesta 1 - carta de control multinomial usando valor-p}

Para probar la homogeneidad de proporciones, se utiliza la prueba chi cuadrado de bondad de ajuste y, según Montgomery y Runger [15] "una forma de reportar los resultados de una prueba de hipótesis es afirmar que la hipótesis nula fue o no rechazada con un nivel de significación especificado", de tal manera que el valor-p definido como "la probabilidad de que el estadístico de prueba asuma un valor que sea al menos tan extremo como el valor observado del estadístico cuando la hipótesis nula HO es verdadera" [15], facilita tomar decisiones sobre el rechazo o no de la hipótesis nula comparando el valor-p con el nivel de significancia, y de manera consecuente, decidir si las muestras tomadas del proceso siguen la distribución multinomial dada por el vector de proporciones nominal; graficar el valor-p de la prueba permite identificar qué tan cierto es que los datos de cada muestra realmente provengan de la distribución dada por la hipótesis nula.

Teniendo en cuenta que bajo $H_{0}$ el valor-p $\sim U[0,1]$ [16], la primera carta de control propuesta es la carta de control para valor-p resultado de la prueba de hipótesis de igualdad de proporciones de una distribución multinomial con respecto a un vector de proporciones nominal. En la carta de control se grafica el valor-p y se compara con un percentil determinado de la distribución uniforme, cuyo complemento delimita la región de rechazo dada por el intervalo $[0, \alpha)$ para un nivel de significancia de $\alpha$ predefinido, así que todos los valores-p que estén en dicha región sugieren la ocurrencia de un cambio en la proporción de al menos una de las categorías. Vea la figura 1.

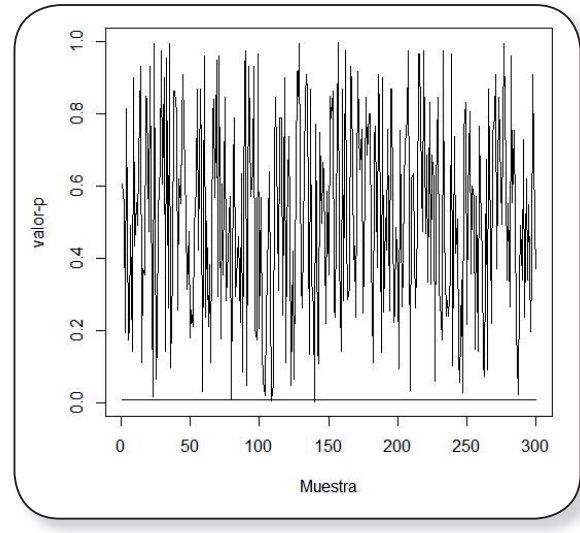

Figura 1. Carta de control usando valor-P Fuente: Autores

2.2 Alternativa Propuesta 1 - carta de control multinomial usando transformación chi cuadrado del valor-p

De manera alternativa, se propone la siguiente carta de control equivalente, usando la transformación chi cuadrado del valor-p.

Considerando que $\quad-2 \ln (x) \sim \chi_{(2)}^{2}$ con $x \sim U[0,1][17]$, la segunda carta de control propuesta toma el valor-p de la prueba de hipótesis de igualdad de proporciones de una distribución multinomial con respecto a un vector de proporciones nominal y lo transforma, y grafica en la carta de control el valor chi cuadrado resultante. De esta manera, por tratarse de una distribución sesgada en el intervalo $[0 ; \infty)$, esta carta de control indicará que el proceso está fuera de control mediante la presencia de puntos en la región de rechazo determinada por $\left(\chi_{(2, \alpha)}^{2} ; \infty\right)$ o por algún patrón de comportamiento no aleatorio. Vea la Figura.2

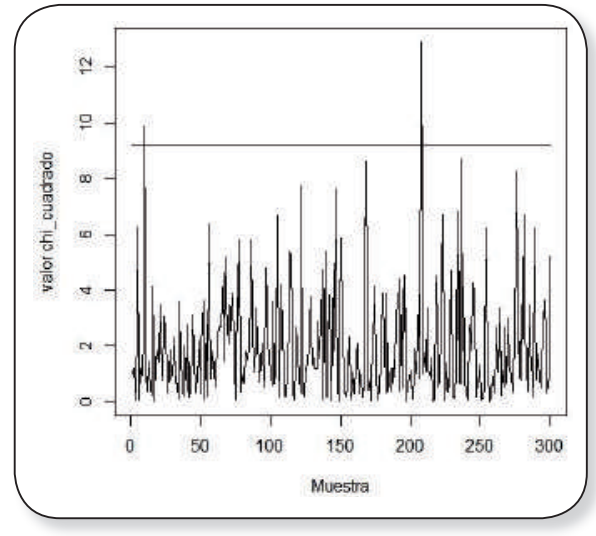

Figura 2. Carta de control usando transformación chi cuadrado del valor-P Fuente: Autores 


\subsection{Propuesta 2 - carta de control multinomial usando aproximación Wilson- Hilferty para variables chi cuadrado}

Utilizando la aproximación Wilson-Hilferty para variables chi cuadrado [17], la segunda propuesta emplea la teoría desarrollada por Shewhart para cartas de control bajo el supuesto de normalidad. Sea $y=\left(\frac{\chi^{2}}{v}\right)^{\frac{1}{3}}$, como $v=2$, los límites de control están dados por (4)-(6).

$$
\begin{aligned}
& L C S=\left(1-\frac{1}{9}\right)+Z_{\alpha / 2}\left(\frac{1}{9}\right) \\
& L C=\left(1-\frac{1}{9}\right) \\
& L C I=\left(1-\frac{1}{9}\right)-Z_{\alpha / 2}\left(\frac{1}{9}\right)
\end{aligned}
$$

De esta manera, al tomar el valor chi cuadrado transformado del valor-p de la prueba de igualdad de proporciones para una variable multinomial, se obtendrá una carta de control para datos individuales, y de esta manera se lleva un proceso multinomial a un proceso normal aproximado, cuya teoría está ampliamente desarrollada y se puede implementar fácilmente con los recursos computacionales requeridos, sin embargo es importante tener en cuenta que esta aproximación mejora cuando el número de grados de libertad tiende a infinito, pero para este caso sólo se consideran 2 grados de libertad de manera invariante. Vea la figura 3.

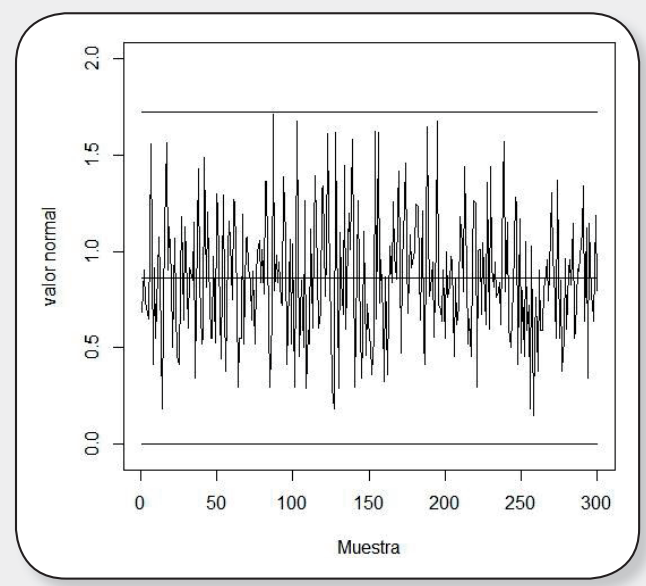

Figura 3. Carta de control usando aproximación Wilson-Hilferty para variables chi cuadrado

Fuente: Autores

\section{Resultados y análisis}

Con el propósito de observar el desempeño de las cartas de control propuestas, es necesario considerar varios escenarios, estudiados vía simulación, realizada bajo la siguiente metodología:

Paso 1: se fija el número de categorías $c=4$

Paso 2: se fija el vector de proporciones en estado estable $\mathrm{v}=(0.80,0.06,0.06,0.08)$

Paso 3: se determina el tamaño de muestra a obtener en cada punto de observación (905)

Paso 4: se fija el número de muestras que se van a observar en el tiempo (300)

Paso 5: para cada corrida se determina si el proceso se rechaza o no; este paso se repite 50.000 veces.

Paso 6: se sacan resúmenes estadísticos de interés

Tomando $c=4$ categorías, las simulaciones se realizaron considerando un proceso en Fase II y considerando incrementos en la primera categoría de $2 \%, 4 \%$ y $6 \%$ en la etapa de control.

Para las simulaciones asumiendo proceso en Fase II, el código está enmarcado en un ciclo for que, para cada repetición, genera 300 vectores de proporciones de acuerdo a la distribución nominal dada y, para cada vector se toma el valor-p de la prueba chi cuadrado de Pearson. En una matriz resumen se almacena el número de rechazos y el número de muestras hasta que ocurre el primer rechazo y, una vez terminadas las repeticiones, se calcula el número promedio de rechazos, el número máximo y mínimo de veces que se rechaza la hipótesis nula y el ARL para cada una de las cartas propuestas.

Para las simulaciones asumiendo proceso fuera de control, la estructura del código es la misma que para el caso anterior, pero las muestras se generan a partir del vector de proporciones modificado de acuerdo a los incrementos ya mencionados. 
No. 2

Julio-Diciembre 2014 ISSN 0122-820X

PP: $93-100$

\subsection{Simulaciones asumiendo proceso en Fase II}

Considere un proceso multinomial con $c=4$ categorías y con vector de proporciones nominal dado por $v=(0.80,0.06,0.06,0.08)$ estable en el tiempo, de donde se toman 300 muestras cada una con tamaño de muestra es $n=905$ [19]. Para cada carta de control se simulan 50.000 corridas y se estudia la distribución de frecuencias del número de rechazos (Re) y la longitud de corrida promedio (ARL). Los resultados se presentan en la Tabla I.

Para las cartas de control multinomial usando valor-p y usando transformación chi cuadrado del valor-p, el número esperado de rechazos obtenido coincide con el teórico, y de esta manera el $A R L=1 / p=1 / \alpha=100, \quad$ sin embargo, para la carta de control multinomial usando transformación Wilson-Hilferty para variables chi cuadrado, el número medio de rechazos es inferior al teórico, de tal manera que la probabilidad de encontrar un punto fuera de control en 300 muestras será 1.42 / $300=0.005$, obteniendo un ARL de 200. De esta manera, la carta de control multinomial usando transformación Wilson-Hilferty para variables chi cuadrado es una carta de control conservadora, ya que no detecta el número de rechazos esperado y por lo tanto indicará que el proceso está bajo control cuando pudiera no estarlo.

Asumiendo control estadístico, la simulación pretende identificar si no hay una alta proporción de falsas alarmas, ya que ésta es una propiedad deseable de las cartas de control. Cuando el proceso está fuera de control, se desea que lo carta detecte rápidamente sin que éstas sean falsas alarmas, esto es, que declare que el proceso está fuera de control cuando realmente no lo está.

\subsection{Simulaciones asumiendo proceso fuera de control}

Considerando nuevamente un proceso multinomial con $\mathrm{k}=4$ categorías y con vector de proporciones nominal dado por $\mathrm{v}=(0.80$,
$0.06,0.06,0.08)$ y que $\sum_{i=1}^{k} p_{i}=1$, el plan

de simulaciones para analizar el desempeño de las cartas de control propuestas bajo condiciones fuera de control, asociadas con el cambio en los parámetros de la distribución, consiste en hacer un cambio porcentual de $2 \%, 4 \%$ y $6 \%$ en la primera categoría de los datos simulados. Para cada carta de control se simulan 50.000 corridas y se analiza la distribución de frecuencias del número de rechazos (Re) y la medida de probabilidad de rechazar la hipótesis nula de homogeneidad de proporciones.

Considerando $\alpha=0.01$, la Tabla I presenta los resultados de las simulaciones de las cartas de control multinomial propuestas para cada escenario. En Fase II, las cartas de control usando valor-p y usando transformación chi cuadrado al valor-p tienen un ARL de 98, mientras que la carta de control multinomial usando transformación Wilson-Hilferty para variables chi cuadrado tiene un ARL de 200. Fuera de control, cuando ocurre un cambio de $2 \%$ o $4 \%$ en la primera categoría, con el respectivo cambio proporcional en las tres categorías restantes, la propuesta 1 y equivalentemente la propuesta 2 , presentan un mejor desempeño que la propuesta 3 . Cuando el cambio es de 6\%, las 3 propuestas presentan un desempeño similar para detectar el cambio, de tal manera que a medida que aumenta el cambio mejora el desempeño de las propuestas.

Tabla I. Comparativo entre propuestas de cartas de control simulando procesos bajo control

\begin{tabular}{|c|c|c|c|c|}
\hline $\begin{array}{c}\text { Incremento en } \\
\text { proporción }\end{array}$ & Criterio & Propuesta 1 & $\begin{array}{c}\text { Alternativa } \\
\text { Propuesta 1 }\end{array}$ & Propuesta 2 \\
\hline \multirow{4}{*}{ Fase II } & Rechazos promedio & 3.05 & 3.05 & 1.42 \\
\cline { 2 - 5 } & Máximo & 13 & 12 & 8 \\
\cline { 2 - 5 } & Mínimo & 0 & 0 & 0 \\
\cline { 2 - 5 } & ARL & 98.42 & 98.34 & 211.02 \\
\hline \multirow{4}{*}{$\%$} & Rechazos promedio & 9.95 & 9.95 & 5.21 \\
\cline { 2 - 5 } & Máximo & 25 & 25 & 16 \\
\cline { 2 - 5 } & Mínimo & 0 & 0 & 0 \\
\cline { 2 - 5 } & P(Re H0) & 0.286 & 0.286 & 0.159 \\
\hline \multirow{4}{*}{$\%$} & Rechazos promedio & 70.95 & 70.95 & 48.63 \\
\cline { 2 - 5 } & Máximo & 102 & 104 & 76 \\
\cline { 2 - 5 } & Mínimo & 44 & 41 & 23 \\
\cline { 2 - 5 } & P(Re H0) & 0.932 & 0.931 & 0.831 \\
\hline \multirow{4}{*}{$6 \%$} & Rechazos promedio & 211 & 211 & 181 \\
\cline { 2 - 5 } & Máximo & 240 & 241 & 213 \\
\cline { 2 - 5 } & Mínimo & 179 & 175 & 146 \\
\cline { 2 - 5 } & P(Re H0) & 1 & 1 & 1 \\
\hline
\end{tabular}

Fuente: Autor 
De acuerdo a lo mencionado anteriormente, a medida que el cambio sea mayor en la primera categoría, es más probable que la hipótesis de homogeneidad de proporciones sea rechazada, sin embargo, la carta de control multinomial usando aproximación Wilson-Hilferty para variables chi cuadrado, provenientes de la transformación del valor-p, presenta un desempeño deficiente comparado con las cartas de control usando valor-p y usando transformación chi cuadrado al valor-p, pues tiene menor habilidad para detectar cambios pequeños, y por lo tanto se recomienda el uso de la carta de control usando valor-p, o equivalentemente, de la carta de control usando transformación chi cuadrado del valor-p.

Se debe tener en cuenta que las cartas de control propuestas no distinguen entre categorías, y los rechazos de la hipótesis solamente sugieren que hubo un cambio en alguna de las proporciones, pero no indica si el cambio está asociado a incrementos o a disminuciones, por lo tanto el analista del proceso deberá recurrir a cartas de control marginales cuando evidencie un comportamiento fuera de control, pues será necesario identificar cuál categoría cambió y cómo lo hizo, con el propósito de plantear un plan de acción correctivo y/o preventivo y mejorar las condiciones de operación del proceso.

\section{Conclusiones}

Se han presentado tres propuestas para monitorear procesos con variables multinomiales mediante cartas de control enmarcadas en el Control Estadístico de Procesos, basadas en el valor-p de la prueba de hipótesis de homogeneidad de proporciones, llamadas carta de control multinomial usando valor-p, carta de control multinomial usando transformación chi cuadrado al valor-p y carta de control multinomial usando transformación Wilson-Hilferty para variables chi cuadrado. Estas propuestas fueron estudiadas vía simulación, analizando la distribución de frecuencias del número de

rechazos (Re) y la longitud de corrida (LR), considerando un escenario bajo control y cambios totales en la distribución de 2\%, 4\% y $6 \%$ en la primera categoría y en las siguientes de manera proporcional, sugiriendo mejor desempeño cuando el proceso está fuera de control la carta de control usando valor-p.

Las cartas de control aquí propuestas deben ser utilizadas para tamaños de muestra grandes, asociadas con procesos de producción masiva como en industrias de alimentos o de confecciones, y se sugiere utilizar la cartas de control marginales cuando sea necesario identificar la categoría que cambió, o cuando se tenga duda sobre si el proceso está o no bajo control. Las restricciones de estas propuestas sugieren un trabajo posterior de profundización, tal como el diseño de cartas de control para procesos con variables multinomiales con tamaños de muestra variable, el diseño de cartas de control con más de 10 categorías, cartas de control para múltiples variables multinomiales, e incluso estudiar el efecto de tamaños de muestra pequeños.

\section{Referencias}

[1] J. Juran, F. Gryna, "Juran's Quality Control Handbook." 4 ed. New York: McGraw Hill, 1988.

[2] D. Montgomery, 2Control Estadístico de la Calidad". Mexico D.F.: Limusa Wiley, p. 789, 2005.

[3] N. Johnson, S. Kotz, "Discrete Multivariate Distributions". New York: Wiley - Interscience. p. 299. 1996. PP: $93-100$

-

Julio-Diciembre 2014 $\mathrm{X}$ 
No. 2

Julio-Diciembre 2014

ISSN 0122-820X

PP: 93-100
[6] J. J. Shiau, C. R. Chen, C. Feltz, "An Empirical Bayes Process Monitoring Technique for Polytomous Data", Quality and Reliability Engineering International, vol. 21, no. 1, pp. 13-28, 2005.

[7] W. Woodall, K. L. Tsui, Y G. R. Tucker, "A Review of Statistical and Fuzzy Quality Control Charts Based on Categorical Data". Frontiers in Statistical Quality Control 5, p. 291, 1997.

[8] C. Spanos, y R. Chen, "Using Qualitative Observations for Process Tuning and Control", IEEE Transactions on Semiconductor Manufacturing, vol. 10, no. 2, pp. 307-316, 1997.

[9] R. Duran, Tesis de Doctorado. Monitoring and Interpreting Multistage and Multicategory Processes Rutgers University, 2009.

[10] T. Raz, J. H. Wang, "Probabilistic and membership approaches in the construction of control charts for linguistic data", Production Planning and Control, vol. 1, no. 3, pp. 147-157, 1990.

[11] H. Taleb, M. Limam, "On fuzzy and probabilistic control charts", International Journal of Production Research, vol. 40, no. 12, pp. 2849 2863, 2002.

[12] H. Taleb, M. Limam, K. Hirota, "Multivariate fuzzy multinomial control charts", Quality Technology $\mathcal{B}$ Quantitative Management, vol. 3, no. 4, pp. 437 - 453, 2006.

[13] H. Taleb, "Control Charts Applications for Multivariate Attribute Processes". Computers $\mathcal{B}$ Industrial Engineering, vol. 56, no. 1, pp. 399 - 410. 2009.

[14] Y. Li, F. Tsung, "Multiple Attribute Control Charts with False Discovery Rate Control", Quality and Reliability Engineering International, vol. 28, no. 8, pp. 857-871, 2012.

[15] D. Montgomery, G. Runger, "Applied Statistics and Probability for Engineers",Nueva York: John Wiley \& Sons, p. 706, 2003.

[16] K. Fairbanks, R. Madsen, "P Values for Test Using a Repeated Significance Test Design”, Biometrika, vol. 69, no. 1, pp. $69-74,1982$.

[17] R. Hogg, A. Craig, "Introduction to Mathematical Statistics", Bogotá: Prentice Hall, X, p. 564, 1995.

[18] E. Wilson, M. Hilferty, "The Distribution of Chi-square", Proceedings of the National Academy of Sciences of the United States of America, vol. 17, no. 12, pp. 684 - 688, 1931.

[19] J. Bromaghin, "Sample Size Determination for Interval Estimation of Multinomial Probabilities", The American Statistician, vol. 47, no. 3, pp. 203 - 206, 1993. 